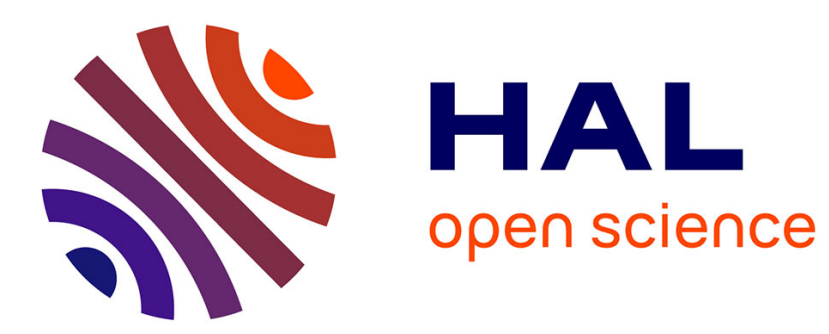

\title{
Les inondations remarquables en France: premiers éléments issus de l'enquête EPRI 2011
}

M. Lang, C. Coeur, A. Bard, B. Bacq, T. Becker, E. Bignon, R. Blanchard, L. Bruckmann, M. Delserieys, C. Edelblutte, et al.

\section{- To cite this version:}

M. Lang, C. Coeur, A. Bard, B. Bacq, T. Becker, et al.. Les inondations remarquables en France: premiers éléments issus de l'enquête EPRI 2011. La Houille Blanche - Revue internationale de l'eau, 2013, 5, p. 37 - p. 47. 10.1051/lhb/2013041 . hal-00936942

\section{HAL Id: hal-00936942 \\ https://hal.science/hal-00936942}

Submitted on 27 Jan 2014

HAL is a multi-disciplinary open access archive for the deposit and dissemination of scientific research documents, whether they are published or not. The documents may come from teaching and research institutions in France or abroad, or from public or private research centers.
L'archive ouverte pluridisciplinaire HAL, est destinée au dépôt et à la diffusion de documents scientifiques de niveau recherche, publiés ou non, émanant des établissements d'enseignement et de recherche français ou étrangers, des laboratoires publics ou privés. 


\title{
Les inondations remarquables en France : premiers éléments issus de l'enquête EPRI 2011
}

\author{
Michel LANG ${ }^{1}$, Denis COEUR ${ }^{2}$, Antoine BARD 2 , Bruno BACQ ${ }^{2}$, Timothée BECKER ${ }^{2}$, \\ Emilie BIGNON ${ }^{2}$, Raphaël BLANCHARD ${ }^{2}$, Laurent BRUCKMANN ${ }^{1}$, Mathieu DELSERIEYS ${ }^{2}$,
} Charlotte EDELBLUTTE 2 , Caroline MERLE ${ }^{2}$

\footnotetext{
1. Irstea, Unité de recherche Hydrologie-Hydraulique - 5 rue de la Doua, 69626 Villeurbanne cedex - michel.lang@irstea.fr

2. Acthys-Diffusion - 233 chemin de Plate-Rousset, 38330 Biviers - denis.cœur@orange.fr
}

\begin{abstract}
RÉSUMÉ. - Un recensement de près de 2000 inondations a été réalisé en 2011 sur l'ensemble du territoire français dans le cadre de l'Evaluation Préliminaire des Risques d'Inondation (EPRI), première phase de la Directive européenne sur les Inondations. En collaboration étroite avec les services de l'Etat concernés, un choix d'environ 300 événements remarquables a ensuite été établi, pour lesquels une description courte (1 à 2 pages) a passé en revue la genèse de l'inondation et ses conséquences. L'article décrit le mode opératoire suivi pour la collecte des données et la sélection des événements puis présente à titre d'illustration dix inondations remarquables (1910, 1930, 1940, 1947-48, 1959, 1980, 1999, 2000-01, 2001 et 2010). Il se termine par les perspectives de valorisation de cette enquête 2011.
\end{abstract}

Mots-clés : Directive Inondation, EPRI, crue historique, inondation remarquable

\section{Significant French Flood Events from the 2011 Preliminary Flood Risk Assessment of the European Flood Directive}

\begin{abstract}
An inventory of about 2000 French flood events has been achieved in 2011, in the framework of the Preliminary Flood Risk Assessment of the European Flood Directive. In collaboration with local authorities, a set of about 300 significant past floods has been documented with a short presentation (1 to 2 pages) of the hydro-meteorological conditions and the adverse consequences. The paper presents the methodological framework for data collection and significant flood selection. Then ten extreme flood events are presented : 1910, 1930, 1940, 1947-48, 1959, 1980, 1999 , 2000-01, 2001 and 2010. Some perspectives are addressed to improve the knowledge of past floods.
\end{abstract}

Key-words: Flood directive, PFRA, historic flood, significant flood

\section{LE CONTEXTE DE LA DIRECTIVE INONDATION}

En introduisant la nécessité de se référer explicitement au passé dans l'évaluation des risques d'inondation, la directive européenne 2007/60/CE du 23 octobre 2007, relative à l'évaluation et à la gestion des risques inondation, engage désormais les autorités publiques à prendre en compte de manière beaucoup plus systématique et structurée les données sur les événements passés, que ceux-ci soient très anciens (plusieurs siècles) ou très récents (quelques mois, quelques années). Le calendrier pour la mise en application de la Directive Inondations (DI) comprend trois étapes, à réactualiser tous les six ans : 1 / fin 2011, l'achèvement de l'Evaluation Préliminaire des Risques d'Inondation (EPRI) suivi de la sélection de Territoires à Risque Important d'inondation (TRI) ; 2/ fin 2013, la cartographie des surfaces inondables et des risques d'inondation pour chaque TRI ; 3/ fin 2015, la mise au point de Plans de Gestion des Risques d'Inondation (PGRI) à l'échelle des districts. Dans ce contexte, la France a décidé de mettre en œuvre une démarche ambitieuse associant collecte, conservation, validation et valorisation de l'information dans le cadre de la création d'une base de données historiques sur les inondations (BDHI). Elle concerne les inondations passées mais également les événements à venir afin que se constitue peu à peu à l'échelle nationale une mémoire référence des inondations.

Le Ministère de l'Ecologie (DGPR) a confié au Cemagref la maîtrise d'œuvre de cette base de données et de la démarche qui l'accompagne. Le programme d'action sur la période 2010-2011 comprenait : a/ la mise au point d'un guide méthodologique pour la collecte des informations historiques sur les inondations ; b/ la confection de la base BDHI ; c/ la collecte des données sur les inondations marquantes du passé sur l'ensemble du territoire français (métropole et départements d'outre-mer, DOM) ; $\mathrm{d} /$ la rédaction de synthèses sur les inondations les plus remarquables en vue de leur intégration dans le rapport EPRI de première étape de la DI ; e/ l'intégration des données des bases existantes et des données collectées dans la BDHI. Nous présentons ici les premiers résultats de l'enquête documentaire menée dans le cadre d'un partenariat disciplinaire entre le Cemagref (hydrologie) et le bureau d'étude Acthys-Diffusion (histoire).

Après avoir décrit le mode opératoire suivi pour la collecte et la sélection des événements, nous présentons à titre d'illustration dix inondations remarquables (1910, 1930, 1940, 1947-48, 1959, 1980, 1999, 2000-01, 2001 et 2010), et nous terminerons par les perspectives de valorisation de 
cette enquête 2011. Les événements retenus couvrent les différents types d'inondation de la DI : remontée de nappe, submersion marine, crues lentes, crues rapides, rupture d'ouvrage.

\section{UNE COLLECTE DE L'INFORMATION EN PLUSIEURS ETAPES}

\section{II.1. Découpage géographique}

La DI s'appuie sur un découpage du territoire français en 14 districts. Il reprend sur la métropole le périmètre des grands bassins hydrographiques, éventuellement redécoupés en cas de bassin transfrontalier : Adour-Garonne, Corse, Escaut-Somme-Côtiers et Sambre (pour Artois-Picardie), Loire-Bretagne, Meuse et Rhin (pour Rhin-Meuse), RhôneMéditerranée, Seine-Normandie. Chaque district est à son tour découpé en unités de présentation (UP). Elles correspondent en général aux sous-bassins, zones littorales ou axes fluviaux remarquables. Leur nombre varie entre 3 et 10 selon l'importance des districts. Certains ne comportent qu'une seule unité de présentation (Corse, Meuse, Sambre). Pour les cinq départements d'outre-mer (Guadeloupe, Guyane, Martinique, Mayotte, Réunion), district et unité de présentation sont confondus.

\section{II.2. Collecte des données}

Pour des raisons pratiques liées à l'agenda général de la DI, il a été convenu que l'enquête documentaire 2011 serait centrée sur la documentation conservée dans les services de l'Etat, plus quelques études référence [Champion, 2001 ; fonds Pardé, http ://iga.ujf-grenoble.fr/communication/parde/] et bases de données régionales. On privilégie de fait une chronologie événementielle contemporaine (le dernier siècle principalement), ce qui n'empêche pas de disposer à travers les documents récents d'informations sur des inondations plus anciennes (XVIII ${ }^{\mathrm{e}}$ et $\mathrm{XIX}^{\mathrm{e}}$ siècles). Le travail de collecte a été réalisé par sept chargés d'étude recrutés pour cette enquête 2011, chacun étant responsable d'un bassin (Adour-Garonne, ArtoisPicardie, Loire-Bretagne, Rhin-Meuse, Rhône-Méditerranée et Corse, Seine-Normandie) ou de l'ensemble des cinq DOM. Ils ont travaillé en étroite collaboration avec les services de l'Etat concernés, chaque DREAL de bassin étant chargée de coordonner la rédaction de la note EPRI.

Un questionnaire préalable a été adressé en janvier aux différents services concernés en vue de préparer le recensement des inondations remarquables, des documents associés et des bases de données régionales. Une réunion de travail a eu lieu ensuite dans chaque DREAL de bassin pour arrêter une première liste de cours d'eau et sites à enjeux et définir les modalités pratiques de récupération des données. D'avril à juillet, les sept chargés d'étude ont sélectionné et numérisé les documents disponibles dans les services.

Ils ont ensuite extrait et synthétisé les informations en trois volets principaux (météorologie, hydrologie, et impacts) en précisant à chaque fois : 1/ la localisation géographique : nom de la localité concernée et éventuellement localisation géographique des zones touchées; 2/ le type d'inondation : débordement de cours d'eau, ruissellement, remontée de nappe phréatique, submersion marine, rupture d'ouvrage de protection, autres (éventuellement) ; 3 / les dates de début/fin de l'évènement et/ ou durée ; 4/ le secteur impacté : étendue de la zone inondée, longueur du cours d'eau ou de la zone côtière ;
5/ la période de retour ; 6/ les conséquences de l'inondation sur : a/ la santé humaine et la société : nombre de décès et nombre de personnes disparues, nombre de personnes évacuées; conséquences en terme de pollution, d'interruption de la fourniture d'eau potable ; services publics mis hors d'usage : écoles, maisons de retraite, hôpitaux, sécurité civile (préfecture, mairie, pompiers, gendarmerie ...) ; b/ l'environnement : pollution de l'eau ou de l'air, dommages sur le sol, la faune et la flore, la biodiversité ; sources de pollution potentielles telles que les installations Seveso, les stations d'épuration; c/ le patrimoine culturel : dommages sur les monuments historiques, les musées, les sites archéologiques, les lieux de culte...; d/ l'activité économique : dommages sur l'habitat (nombre de logements inondés), les infrastructures (routes, voies ferrées, aéroports, centrales électriques...), l'activité agricole (élevage, cultures, forêts, extraction minière, pêche), l'activité économique hors agricole (usines, construction, services, commerces...).

Il était prévu initialement de scanner l'ensemble des documents disponibles dans les services, de saisir dans la base BDHI les informations génériques de chaque inondation (lieu, date, type d'inondation, classe de l'événement intensité et dommages), et de faire ainsi ressortir ensuite les événements les plus remarquables. Devant l'ampleur de la tâche et compte tenu d'un calendrier de collecte très contraint (six mois), le mode opératoire a été modifié. Après une première analyse des documents de synthèse disponibles sur les inondations du passé (cf. volet historique des PPRi, atlas de zones inondables, RIC des Services de Prévision des Crues, études référence ...) et des échanges avec les DREAL, cinq à dix événements majeurs ont été sélectionnés par unité de présentation. Différents critères ont présidé à ce choix : le type d'événement (débordement de cours d'eau, remontée de nappe, ruissellement en versant, submersion marine, rupture d'ouvrage), ses caractéristiques hydro-météorologiques (intensité ou extension spatiale), ses conséquences socio-économiques, mais également le fait qu'il constituait ou non un phénomène référence dans les documents officiels (PPRi, AZI ...) ou qu'il était le dernier événement majeur en mémoire. Les informations ont alors été recherchées uniquement sur la liste des inondations sélectionnées.

En complément de cette enquête, un travail parallèle a été mené dans le fonds "Inondations" des Archives Nationales (grandes études de référence). Des échanges ont eu lieu également avec des équipes universitaires ayant travaillé sur la thématique des inondations historiques, en particulier les laboratoires GEODE à Toulouse, GESTER à Montpellier, LSCE Gif-sur-Yvette et CRHQ-Université de Caen. Le recensement des inondations en zone de montagne a aussi exploité les bases de données des services RTM (http ://rtm-onf.ifn.fr/). Celles-ci rassemblent sur les 11 départements alpins et pyrénéens concernés des informations sur plus de 30000 événements passés (affaissement, avalanche, chute de blocs, crue torrentielle, glissement de terrain, inondation, ravinement, tassement). L'identification des submersions marines a pu être complétée par l'apport de bases de données existantes. Enfin, un travail spécifique a été réalisé en 2011 par le BRGM à partir de la base tsunami (http ://www.tsunamis.fr/) en vue d'extraire les informations relatives aux submersions marines, soit au final 33 événements sur la période 16941940 retenus initialement comme faux tsunamis, décrit comme "raz-de-marée " dans la littérature et correspondant en fait à des submersions liées à des tempêtes et/ou des épisodes de forte pluie. 


\section{II.3. Synthèse sur les événements remarquables}

La structure générale des rapports EPRI pour la Directive Inondation comporte trois parties : $1 /$ une introduction sur la directive inondations et la phase EPRI ; 2/ une présentation $\mathrm{du}$ district hydrographique (géographie, types d'inondation, nature des enjeux, politique de gestion du risque) ; 3/ une évaluation des conséquences des inondations (inondations marquantes du passé, impacts potentiels des inondations futures), à l'échelle du district puis de chacune des unités de présentation.

Le plan type sur la description des inondations marquantes du passé comporte une description rapide d'événements de référence choisis pour illustrer chacune des typologies d'inondation rencontrée dans le district : localisation schématique de l'extension spatiale sur la carte du district et résumé des caractéristiques hydro-météorologique de l'événement et de ses conséquences. On trouve ensuite une présentation de cinq à dix inondations marquantes par unité de présentation : tableau et carte de localisation des cours d'eau et secteurs d'intérêt sur lesquels des informations seront fournies, tableau des inondations marquantes (régime hydroclimatique, type de submersion, lieu, date) et texte descriptif d'une à deux pages. Celui-ci aborde successivement la genèse de l'événement, la description de l'aléa (cumuls et ou intensités pluviométriques, cotes d'eau et débits au droit de stations limnimétriques, hauteurs de submersion, informations diverses sur le vent, la neige, des embâcles-débâcles éventuelles, etc.), et les conséquences de l'événement en terme de victimes, dommages, perturbation à l'activité socioéconomique. Un tableau récapitulatif résume en quelques lignes les caractéristiques de l'événement : particularités hydro-météorologiques (genèse, intensité), zones inondées, impacts. Une à deux illustrations sont fournies pour chaque événement pour illustrer par exemple la répartition spatiale du cumul des précipitations, l'hydrogramme de la crue, les conditions de submersion ou les dommages après la catastrophe, etc. En annexe de chaque rapport figure un tableau chronologique général des inondations recensées sur chaque unité de présentation : cours d'eau, localisation, date, type d'inondation, hydrométrie et pluviométrie, impacts (pertes humaines, dommages), crue de référence (PPRi, AZI), commentaire éventuel. Un tableau spécifique des submersions marines est également produit le cas échéant : localisation, date, vent, marée, niveau, commentaire.

\section{PRESENTATION D'UNE DIZAINE D'EVENEMENTS REMARQUABLES}

La collecte des données réalisée entre mars et juillet 2011 a abouti à un listing de près de 2000 inondations. Quelque 300 événements plus remarquables ont été sélectionnés et ont fait l'objet d'une description synthétique (une à deux pages). Le volume complet des rapports produits pour les 14 districts avoisine 900 pages dont 550 pages pour la description des événements et 350 pages de tableaux chronologiques annexes listant les inondations. Après la phase d'urgence de 1'EPRI 2010-2011, une lecture transversale détaillée de l'ensemble de ces informations est à réaliser. Pour illustrer cet important travail d'enquête 2011, nous présentons ci-dessous, de manière synthétique par rapport au rendu de l'EPRI, dix événements particulièrement remarquables.

\section{III.1. Crue généralisée de janvier 1910}

Le débordement de la Seine de janvier et février 1910 est un événement majeur tant par son intensité que par les dégâts engendrés. La fin de l'année 1909 est très humide avec $450 \mathrm{~mm}$ de précipitations sur trois mois. Le mois de janvier 1910 est caractérisé par des précipitations de pluie et de neige abondantes sur l'ensemble du bassin de la Seine, avec un cumul des précipitations de l'ordre de $300 \mathrm{~mm}$ dans la partie haute du bassin versant, de $110 \mathrm{~mm}$ dans la partie centrale, et de $280 \mathrm{~mm}$ sur la Seine aval. Les pluies du 18 au 21 janvier ruissellent du fait des sols saturés. L'onde de crue se développe sur la Seine, mais également sur la Marne. Deux autres se forment sur l'Yonne. Les pics de la Seine amont et de l'Yonne se rejoignent et sont à l'origine d'une crue très importante sur la région parisienne. La plupart des cours d'eau connaissent un événement de période de retour centennale ( $T=100 \mathrm{ans}$ ) avec des développements et des conséquences différents d'un secteur à l'autre du bassin, sans compter l'influence de la mer en Seine Aval. Malgré son ampleur, l'événement de janvier 1910 n'est pas le plus important en cote : en février 1658, la hauteur de la Seine aurait atteint $8.96 \mathrm{~m}$ au Pont d'Austerlitz à Paris, soit un peu plus de $30 \mathrm{~cm}$ au-dessus du niveau de 1910. La comparaison en débit est plus délicate du fait de changements importants sur la capacité d'écoulement du cours canalisé de la Seine.

Tableau 1 : Liste d'événements sélectionnés

\begin{tabular}{|l|l|}
\hline Evénement & Districts concernés \\
\hline Crue généralisée de janvier 1910 & Rhin, Rhône-Méditerranée, Seine-Normandie \\
\hline Crue du Tarn et de la Garonne du 3-5 mars 1930 & Adour-Garonne \\
\hline Crue du Tech, de la Têt et de l'Agly du 16-20 octobre 1940 & Rhône-Méditerranée \\
\hline Crue de décembre 1947 - janvier 1948 sur le Nord-Est de la France & Meuse, Rhin \\
\hline Rupture du barrage de Malpasset le 2 décembre 1959 & Rhône-Méditerranée \\
\hline Cyclone Hyacinthe à la Réunion du 18 au 27 janvier 1980 & Réunion \\
\hline Crue de l'Aude du 12-13 novembre 1999 & Rhône-Méditerranée \\
\hline Crues en Bretagne de décembre 2000 - janvier 2001 & Loire-Bretagne \\
\hline $\begin{array}{l}\text { Remontées de nappe et inondations de mars-avril 2001 dans le Nord } \\
\text { de la France }\end{array}$ & Artois-Picardie, Seine-Normandie \\
\hline Tempête Xynthia du 27-28 février 2010 & Adour-Garonne, Loire-Bretagne \\
\hline
\end{tabular}



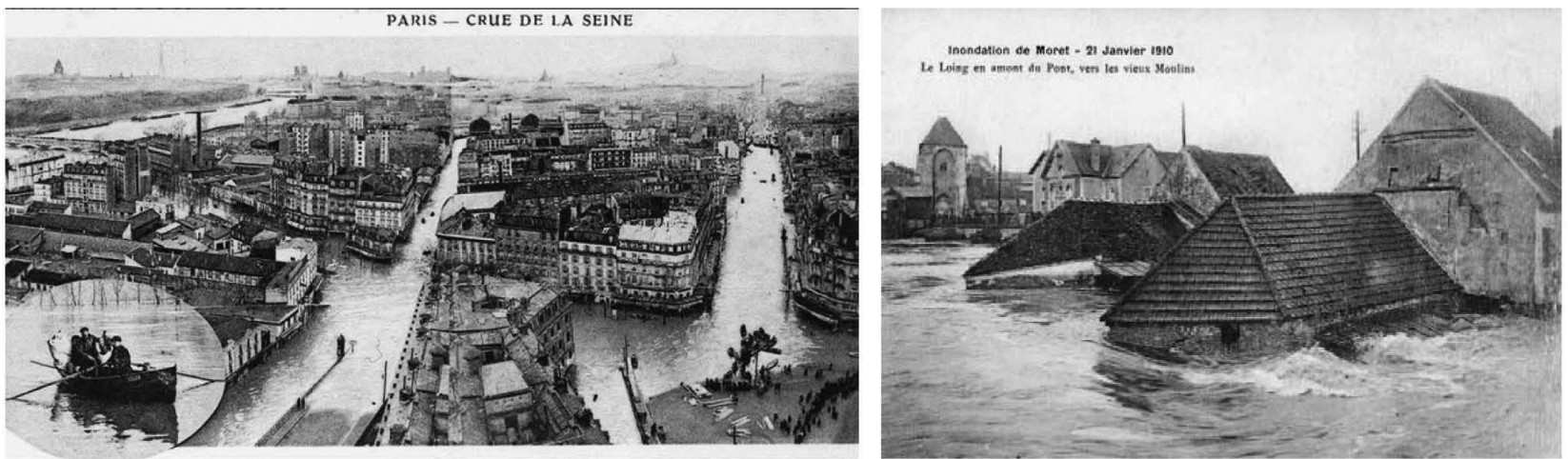

Figure 1 : Inondations de janvier 1910: (a) perspective depuis la tour de l'horloge Gare de Lyon à Paris (29 janvier); (b) Loing à Moret-sur-Loing (source : crue1910..fr).

Le bilan de la crue sur le bassin de la Seine est catastrophique, avec l'inondation de plusieurs milliers de maisons, immeubles, infrastructures, industries, et un arrêt d'activité pendant plusieurs semaines. Le nombre de sinistrés atteint 150000 sur la région parisienne. Le total des dommages est estimé à 400 millions de francs-or, soit plus de 1.6 milliards d'euros (2009). L'événement hydrométéorologique a également fortement touché l'est de la France, en particulier les affluents du Rhin dans le sud de l'Alsace. La plaine de l'Ill est totalement inondée de Colmar au sud de Strasbourg. On relève de nombreuses ruptures de digues. Les activités humaines et économiques sont fortement impactées. Même scénario sur le bassin du Doubs, avec plus de $200 \mathrm{~mm}$ du 18 au 21 janvier sur un épais manteau neigeux qui entre rapidement en fusion. Les activités industrielles sont fortement impactées sur tout le secteur. C'est la seule inondation connue qui a submergé les quais de Besançon et envahi toute la boucle du centre ville.

\section{III.2. Crue du Tarn et de la Garonne du 3-5 mars 1930}

L'événement concerne principalement le bassin du Tarn et celui de la Garonne en aval d'Agen. A partir du
28 février 1930, un anticyclone se positionne entre les Iles Britanniques et les Balkans, alors qu'une dépression est centrée sur la péninsule ibérique. Du $1^{\text {er }}$ au 3 mars le système génère un flux doux et humide de sud-est en provenance de Méditerranée qui balaye le sud-ouest de la France et vient buter contre les reliefs du Massif Central. La violence de la crue s'explique par un ensemble de facteurs. Les pluies n'ont cessé depuis l'automne précédent et à partir de janvier une bonne partie des sols est saturée. Les cumuls de précipitations sont très importants. D'octobre 1929 à février 1930, on relève $1177 \mathrm{~mm}$ à Lodève, $840 \mathrm{~mm}$ à Florac et $533 \mathrm{~mm}$ à Castres (pour des moyennes climatologiques respectivement de 541, 474 et $312 \mathrm{~mm}$ ). Du $1^{\text {er }}$ au 3 mars les cumuls de précipitations dépassent $200 \mathrm{~mm}$ sur un territoire allant de la Montagne Noire au sud du Larzac et jusqu'au sud-ouest de Castres. Par ailleurs, en montagne, la remontée des températures associée aux fortes pluies entraîne une fusion très rapide du manteau neigeux (de 20 à $100 \mathrm{~cm}$ ) encore présent au-dessus de $600 \mathrm{~m}$. Le Tarn atteint la cote de $9.1 \mathrm{~m}$ à Albi et $21.5 \mathrm{~m}$ à Saint-Sulpice (confluence avec l'Agout), pour un débit maximum estimé à $6500 \mathrm{~m}^{3} / \mathrm{s}(T=100$ ans). Les hauteurs d'eau record de la crue de 1875 sur la Garonne sont par endroits dépassées en aval de la confluence avec le Lot.
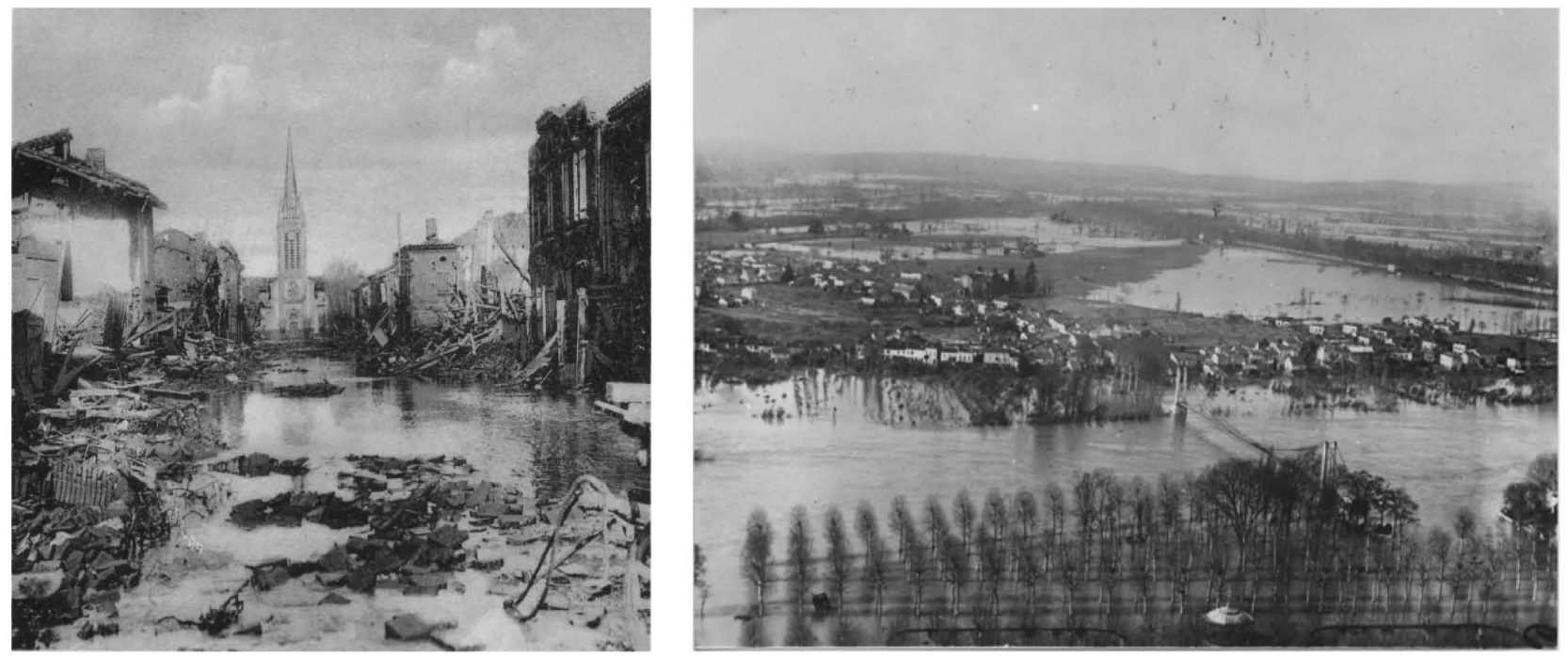

Figure 2 : Crue de mars 1930 : (a) Le village de Reynies après le passage des eaux; (b) Agen à la confluence entre la Garonne et le Tarn (sources: DDT82 et Aquadoc). 
Le bilan est très lourd, avec au total 210 morts, et plus particulièrement à Moissac et Montauban (resp. 100 et 25 morts). On dénombre environ 10000 sinistrés, près de 3000 maisons détruites ainsi que des dizaines d'ouvrages d'art. Les dommages sont estimés à plus d'un milliard de francs (valeur 1930). Le Président de la République, Gaston Doumergue vient en visite sur les lieux et fait voter des lois de solidarité pour l'indemnisation des victimes et la réparation des dommages.

\section{III.3. Crue du Tech, de la Têt et de l'Agly du 16-20 octobre 1940}

Les crues qui touchent la moitié occidentale des CôtiersOuest entre le 16 et le 20 octobre 1940 sont provoquées par une averse extensive méditerranéenne centrée sur le massif du Canigou. Les précipitations sont d'une intensité, d'une extension spatiale et d'une durée exceptionnelle. Des cumuls records sont enregistrés à Saint-Laurent-en-Cerdans sur le flanc sud du Canigou : $1000 \mathrm{~mm}$ le 17 et $1930 \mathrm{~mm}$ entre le 16 et le 20 octobre. La montée des eaux est extrêmement rapide sur les cours d'eau concernés, trois mètres en moins d'une demi-heure pour le Tech à Amélie-les-Bains. On estime le débit maximum atteint par la rivière en ce point à au moins $3000 \mathrm{~m}^{3} / \mathrm{s}(150<T<400$ ans $)$. Le débit de l'Agly à Rivesaltes atteint $2000 \mathrm{~m}^{3} / \mathrm{s}$ (bassin versant de $1070 \mathrm{~km}^{2}$ ), et celui de la Têt à Perpignan $3300 \mathrm{~m}^{3} / \mathrm{s}$ (bassin versant de $1580 \mathrm{~km}^{2}$ ) avec une hauteur d'eau de $5.60 \mathrm{~m}$. Les crues balayent les lits, emportant berges, constructions, troncs d'arbres, etc. Le transport solide est très important. On estime que le lit du Tech s'est rehaussé en moyenne d'environ $70 \mathrm{~cm}$ entre 1923 et 1942 essentiellement du fait des apports de la crue de 1940. De même, l'érosion intense des berges alluvionnaires provoque un élargissement considérable de la bande active. Sur certains tronçons, elle atteint la centaine de mètres. Au défilé de Baillanouse, trois kilomètres à l'aval de Prats-de-Mollo, un éboulement gigantesque barre la vallée d'un mur de 40 mètres de haut sur 200 mètres de long.

La vallée du Tech est la plus impactée par la catastrophe. La gare d'Amélie-les-Bains est rasée ainsi que deux usines électriques, plus une autre à la Coumelade.
Une soixantaine d'immeubles sont détruits à Arles-surTech et Amélie-les-Bains, une dizaine à Prats-de-Molo. Plusieurs dizaines de petits ponts sont broyés par les flots. Plus en aval, la plaine d'Argelès-sur-mer est noyée sous un mètre d'eau. En amont du pont de Brouilla, le Tech occupe un lit de $400 \mathrm{~m}$ de large (50 m en temps normal). Au total, des milliers d'hectares de terres agricoles sont emportées, ravinées, ou recouvertes sous un épais dépôt de sédiments. L'évènement touche également les autres bassins côtiers, Agly, Têt jusqu'à celui de l'Orb, ainsi que le bassin versant de l'Aude et les vallées limitrophes côté espagnole. A Olette, la Têt en crue fait écrouler le mur de soutènement de la $\mathrm{RN}$ et de l'avenue de la gare. La voie ferrée est emportée au niveau de la confluence avec le Cabrils. A Prades, le pont de la RN116 est détruit. On dénombre en tout 50 morts en France (dont la moitié à Amélie-les-Bains et ses environs) et plus de 300 morts en Catalogne.

\section{III.4. Crue de décembre 1947 - janvier 1948 sur le Nord-Est de la France}

Fort enneigement, redoux et pluies exceptionnelles sur le Nord-Est de la France sont à l'origine de la crue majeure de décembre 1947 qui a touché une grande partie du bassin Rhin-Meuse. Le réchauffement à l'origine de la fonte des neiges a été plus intense qu'en 1919, mais c'est surtout le cumul des précipitations en cinq jours sur la partie centrale du massif vosgien qui est remarquable : $415 \mathrm{~mm}$ à Wildenstein (haut-bassin de la Thur), avec un maximum journalier de $185.5 \mathrm{~mm}$ sur ce site. La période de retour de la crue est au moins centennale sur la Meuse amont et médiane, la Meurthe, la Moselle et les affluents alsaciens du Rhin. De nombreux dommages sont recensés en Lorraine et en Alsace. On comptabilise 600 sinistrés dans la vallée de la Meuse, 18000 sinistrés et 2000 immeubles endommagés à Nancy, trois décès et environ un milliard de francs de l'époque de dommages sur le Haut-Rhin. Le contexte d'après-guerre renforce encore la gravité de la situation matérielle. On relève à l'époque l'usage des expressions " sinistrés de la guerre " et "sinistrés des inondations du 29 et 30 décembre 1947 ».

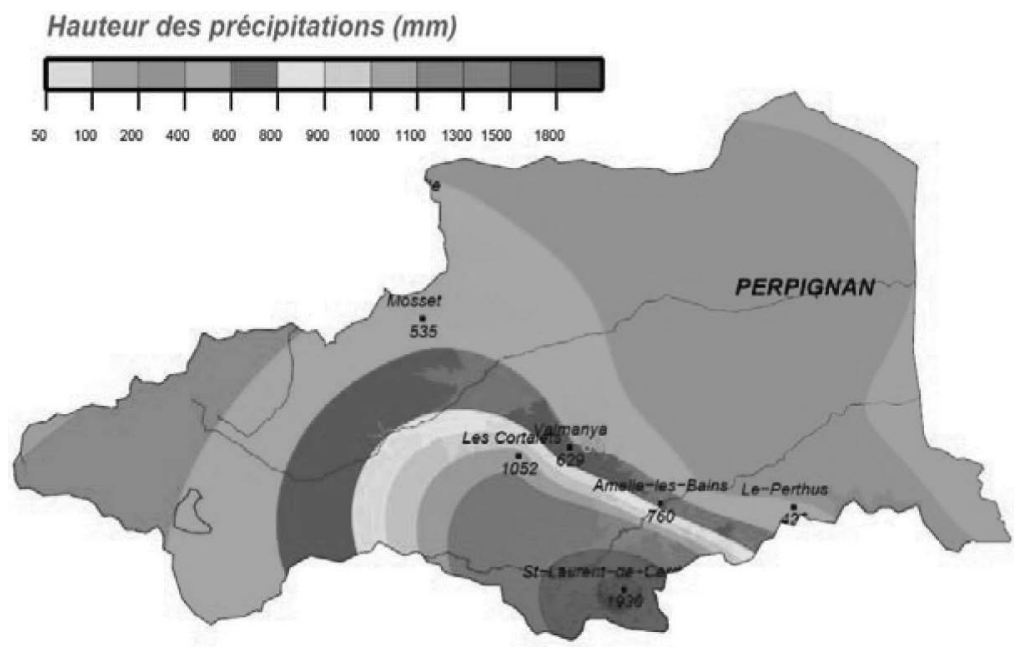

Figure 3 : Cumul pluviométrique sur la période 16-20 octobre 1940 dans les Pyrénées orientales (source : Météofrance, Pluies Extrêmes, v. 11 mars 2011). 

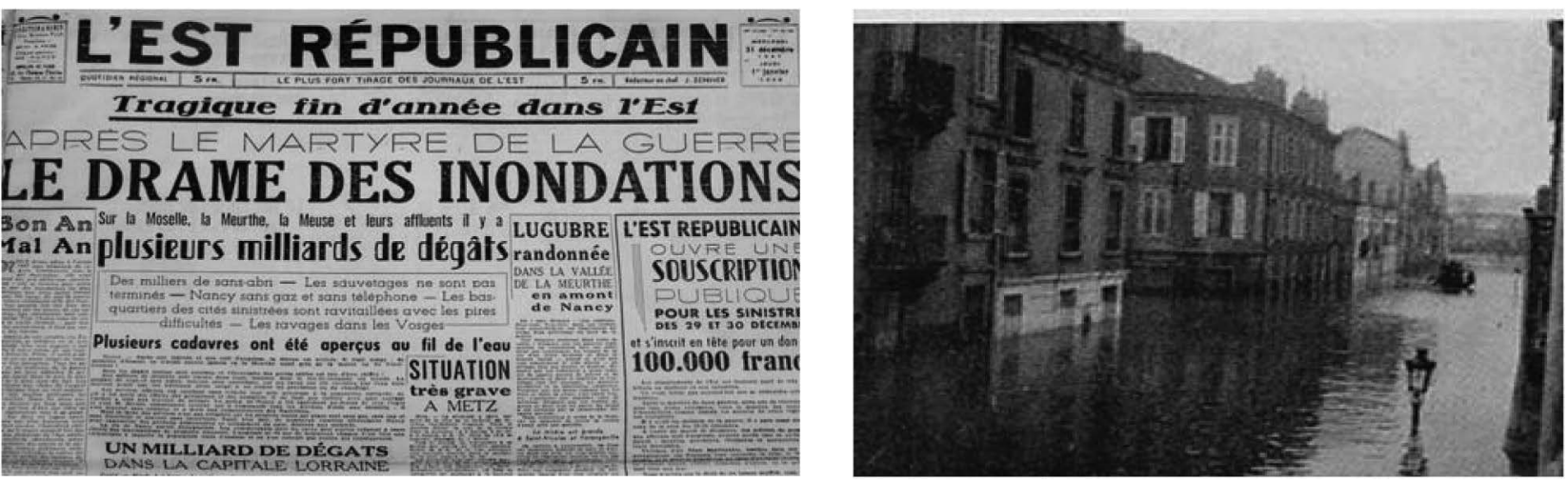

Figure 4 : (a) l'Est Républicain, 31/12/1947-01/01/1948; (b) rue Eugène Vallin à Nancy, déc.1947 (Météo France, Pluies Extrêmes).

\section{III.5. Rupture du barrage de Malpasset le 2 décembre 1959}

Construit entre 1952 et 1954 dans la vallée du Reyran, une dizaine de kilomètres en amont de Fréjus, le barrage de Malpasset répond au besoin crucial d'approvisionnement en eau potable et d'irrigation de la région. De type voûte (largeur $250 \mathrm{~m}$, hauteur $60 \mathrm{~m}$ ), sa capacité de stockage est de 50 millions de $\mathrm{m}^{3}$. Les circonstances de la catastrophe sont aujourd'hui bien connues. Les trois dernières semaines de novembre 1959 ont été particulièrement pluvieuses, avec des cumuls de plus de $400 \mathrm{~mm}$ à Fréjus. Les fortes précipitations méditerranéennes de fin novembre-début décembre provoquent une crue très importante de l'Argens (presque centennale dans la basse plaine de l'Argens, la plus forte survenue au cours du $\mathrm{XX}^{\mathrm{e}}$ siècle), bien inférieure tout de même à l'événement de juin 2010. Le niveau de la retenue du barrage de Malpasset s'élève très rapidement, plus de quatre mètres au cours des $24 \mathrm{~h}$ précédant la rupture, alors que pour des raisons techniques et judiciaires, les tests habituels de solidité n'ont pas été réalisés. En aval, la présence du chantier de l'autoroute Marseille-Nice ne permet pas d'effectuer de vidange. Le remplissage rapide augmente alors les phénomènes de pression sur les roches (gneiss) sous-jacentes, qui conduisent à désolidariser l'ancrage du barrage en rive gauche.
Le 2 décembre au soir, l'ouvrage cède libérant d'un coup un volume de 50 millions de $\mathrm{m}^{3}$ d'eau qui dévale la petite vallée du Reyran à une vitesse estimée à $10 \mathrm{~m} / \mathrm{s}$. Tout est emporté, les habitations riveraines sont rasées et environ 1350 ha de terres cultivées sont ravagés. La vague atteint en quelques minutes la plaine de Fréjus qu'elle submerge d'eau et de boue. Au total, on dénombre 423 victimes et environ 7000 sinistrés. Routes, téléphone, électricité et eau sont coupés. La N7 et la voie ferrée sont détruites sur plusieurs centaines de mètres, de nombreuses terres agricoles et la base aérienne sont ravagées. Fréjus est sinistrée, isolée, et une couche de boue de $50 \mathrm{~cm}$ d'épaisseur recouvre une grande partie de la ville. La prise de conscience engendrée par cette catastrophe entraînera notamment la mise en place d'un "Comité technique permanent des Barrages " ayant autorité sur l'étude et la critique de projets et le contrôle de l'exécution des ouvrages.

\section{III.6. Cyclone Hyacinthe à la Réunion du 18 au 27 janvier 1980}

Le cyclone Hyacinthe est passé par trois fois à proximité de la Réunion entre le 18 et le 27 janvier 1980, entretenant la perturbation 14 jours durant. La pluviométrie enregistrée durant cette période est exceptionnelle

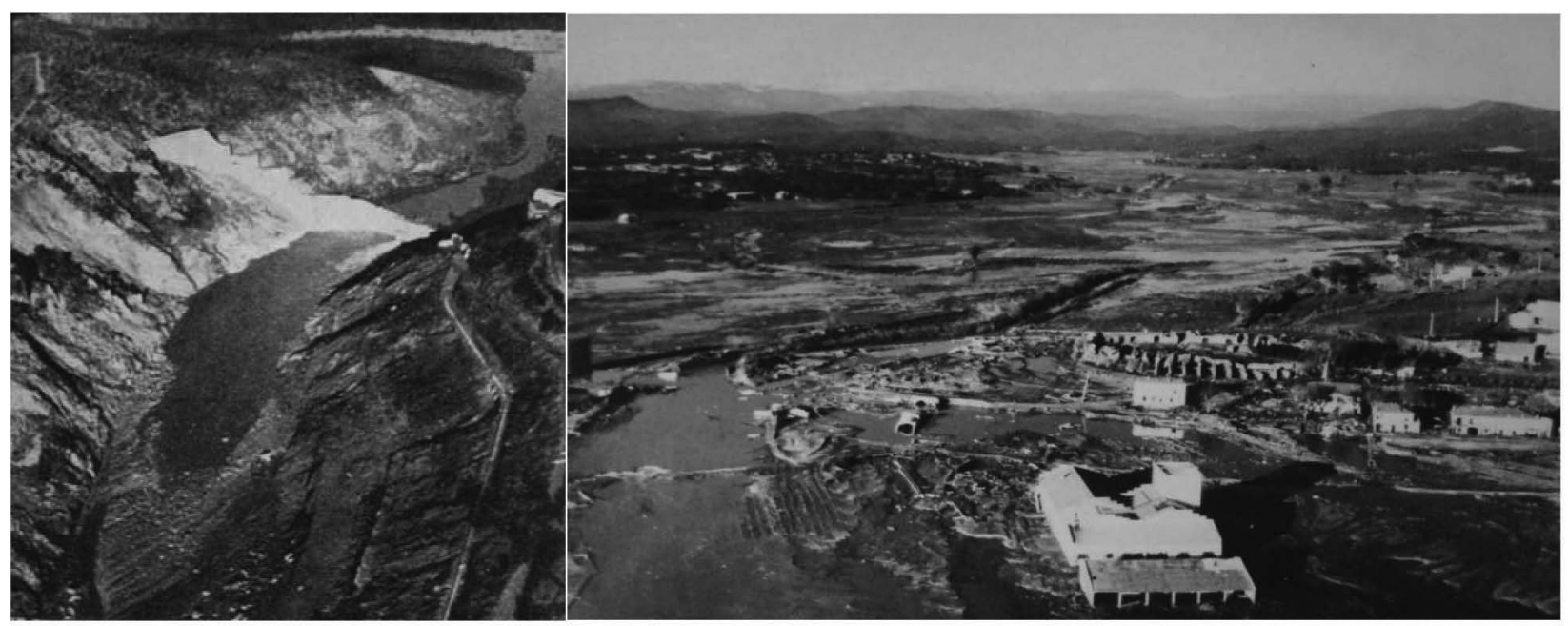

Figure 5 : Rupture du barrage de Malpasset le 2 décembre 1959: a/ le barrage juste après sa rupture ; b/ la plaine de Fréjus juste après le passage des eaux (source : Musée local de Fréjus). 

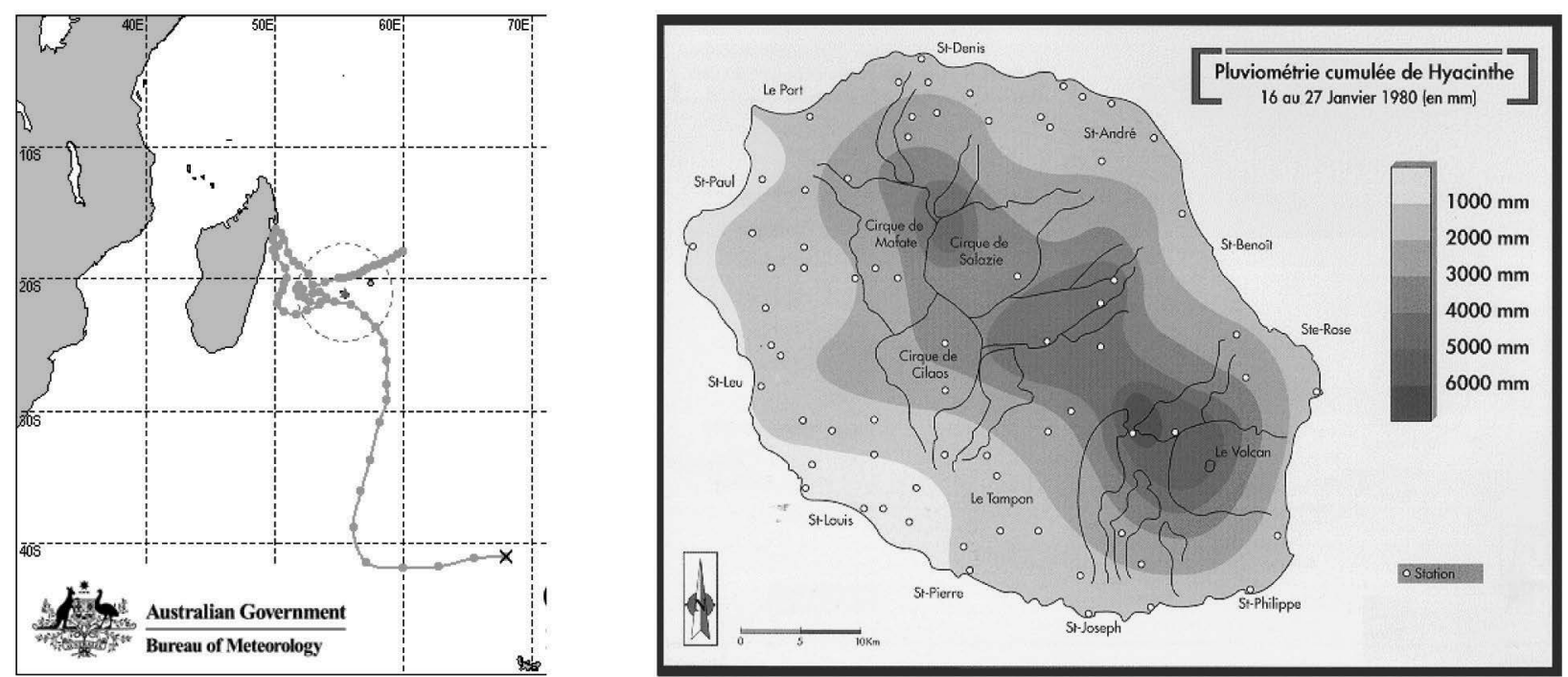

Figure 6 : Cyclone Hyacinthe : (a) trajectoire (en violet, rayon de $400 \mathrm{~km}$ autour de la Réunion) ; (b) cumul pluviométrique du 16 au 27 janvier 1980 (sources : Bureau of Meteorology, Australie et DDE).

sur la majorité de l'île : $1742 \mathrm{~mm}$ en 24 h à Grand Ilet, $1090 \mathrm{~mm}$ à Commerson. Les cumuls les plus importants dépassent cinq à six mètres sur 11 jours ! La Réunion possède avec cet événement les records mondiaux de précipitations de $72 \mathrm{~h}$ à 15 jours avec des cumuls sur 5,10 et 15 jours respectivement de 4979,5678 et $6083 \mathrm{~mm}$ (http ://nws.noaa.gov/oh/hdsc/record_precip/record_precip_world.html), et plus récemment du cyclone Gamède du 24 au 27 février 2007 (cumuls sur 3 et 4 jours respectivement de 3929 et $4869 \mathrm{~mm}$ ). En-dehors du littoral, les pluies sont rapidement supérieures à deux mètres et augmentent vers l'intérieur des terres. Les secteurs les plus arrosés sont les régions de Commerson $(6401 \mathrm{~mm})$ et de Grand Ilet (5 $237 \mathrm{~mm})$ sur les hauteurs de l'île. D'une manière générale, la côte est et la zone centrale ont été les plus arrosées. Ces cumuls extraordinaires sont à l'origine de nombreuses inondations, d'autant que la saturation des sols amplifie encore les ruissellements. Les ravines sont très rapidement saturées et génèrent d'importants débordements. Les débits de pointes enregistrés sont remarquables et sont les premiers facteurs de dommages, le vent n'ayant pas été trop violent.

Le passage de Hyacinthe cause la mort de 25 personnes, emportées par les eaux, et sinistre les habitations de 8000 personnes. Au total, 288 habitations sont détruites et 1712 sont endommagées. Les dommages aux réseaux et aux cultures sont également énormes. Une estimation (1980) fait état d'un montant de 676 millions de francs de dégâts.

\section{III.7. Crue de l'Aude du 12-13 novembre 1999}

Le phénomène cévenol qui frappe à la mi-novembre 1999 les départements de l'Aude, de l'Hérault, des PyrénéesOrientales et du Tarn est d'une ampleur remarquable tant par l'intensité des pluies que par son extension spatiale. On enregistre une lame d'eau de $200 \mathrm{~mm}$ sur une bande de plus de $7000 \mathrm{~km}^{2}$, avec des maxima extrêmes supérieurs à $600 \mathrm{~mm} /$ jour $(624 \mathrm{~mm}$ à Lézignan-Corbières le 12 novembre). Des surcotes marines de plus d'un mètre, liées à des houles d'est et des vents violents (jusqu'à

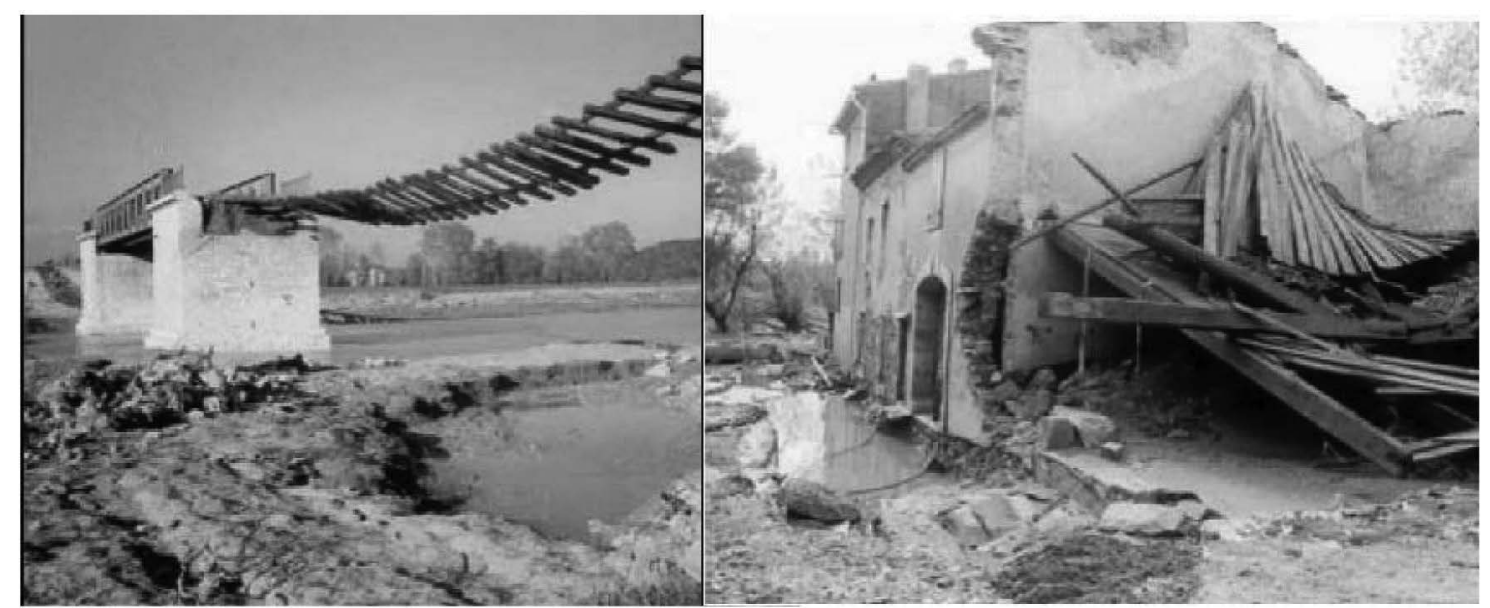

Figure 7 : Crues du 12 et 13 novembre 1999 sur le bassin versant de l'Aude : (a) remblai de la voie ferrée emporté par la crue de l'Aude à Sallèle ; (b) maison à Luc-sur-Orbieu après le passage de la crue de l'Orbieu (source : EGIS Eau, rapport AZI). 
$140 \mathrm{~km} / \mathrm{h}$ ), viennent aggraver les inondations, notamment à proximité des embouchures de l'Aude et de l'Agly. Sur les secteurs amont, les cours d'eau se transforment en torrents entraînant tout sur leur passage et créant d'importants embâcles. Sur les basses plaines de l'Aude, l'inondation de plaine est aggravée par la rapidité de montée du niveau des eaux à l'amont associée aux ruptures d'ouvrages (canal de navigation, remblai SNCF). Les ruissellements intenses génèrent également des inondations pluviales dans les secteurs périurbains et urbains (cf. VilleneuveMinervois). Outre l'Aude (3000 m³ à Moussoulens) et l'Agly (2 $110 \mathrm{~m}^{3} / \mathrm{s}$ à Rivesaltes), la Berre (Corbières), le Thoré et l'Agout sur le haut bassin du Tarn sont particulièrement affectés. La période de retour de la crue est plus que centennale sur la Berre, le Verdouble, l'Argent-Double, la Clamoux, le Lauquet et l'Orbieu.

Les écoulements ravinent les routes, emportent les ponts et les lignes de chemins de fer. Les coulées de boue envahissent les habitations. Les cultures subissent des destructions sans précédent. Un millier d'hectares de vignes disparaît sous les eaux. La zone industrielle de la Molière-Basse, située à la confluence de trois rivières torrentielles est également ravagée. Le bilan humain est lourd : 35 morts, dont 25 dans l'Aude, plus un disparu. Près de la moitié des victimes périssent dans leur véhicule ou à proximité. Sans l'intervention des secours par bateaux et hélicoptères (600 hélitreuillages sont réalisés), le bilan aurait été encore bien plus sévère. A noter de nombreux glissements de terrain dont l'un ensevelit une maison et quatre personnes dans le Tarn. Le bilan matériel est évalué provisoirement à 3.5 milliards de francs à peu près également répartis entre les particuliers (habitations et véhicules) les activités économiques (agriculture, commerces et industries) et les équipements publics. Quatre cent trente-huit communes sont sinistrées.

\section{III.8. Crues en Bretagne de décembre 2000 - janvier 2001}

$\mathrm{Au}$ cours de l'hiver 2000-2001, un vaste système dépressionnaire couplé à un courant d'ouest d'altitude s'installe en Atlantique-Nord générant en Bretagne une succession d'épisodes pluvieux quasi ininterrompue du 18 septembre 2000 à la fin janvier 2001. C'est l'hiver le plus humide qu'ait connu la région depuis l'enregistrement continu des données, avec des cumuls sur six mois égaux ou supérieurs à la pluviométrie annuelle moyenne (entre 740 et $1300 \mathrm{~mm}$ ). Il s'en suit une série de quatre épisodes de crues remarquables.
Les inondations de la mi-novembre 2000 en Ille-etVilaine. Les cumuls de précipitations pour le mois d'octobre 2000 en Bretagne correspondent à deux fois la moyenne mensuelle. La séquence pluvieuse des 11 et 12 novembre (resp. 40 et $60 \mathrm{~mm}$ ) génère les premières crues sur les bassins nord-est de la région ( $T \approx 20$ ans). A Rennes l'Ille, proche de la crue centennale, inonde la ville.

Inondations de la mi-décembre 2000. Les pluies s'abattent cette fois le 11 et le 12 décembre sur l'ouest de la Bretagne avec des cumuls sur 30 heures compris entre 80 et $110 \mathrm{~mm}$. Les crues les plus fortes sont observées sur les bassins de l'Odet et la Laitia ( $T \approx 100$ ans), puis sur l'Aulne, le Jarlot, la Scorff et la Blavet $(20<T<50$ ans $)$, et de façon plus modérée sur le bassin de la Vilaine. Les agglomérations de fond d'estuaire (Quimperlé, Pont-Aven, Quimper, Châteaulin, Landerneau, Morlaix) affrontent au même moment des marées de grande amplitude (coefficient de 99), ce qui les rend doublement vulnérables.

Inondations du $1^{\text {er }}$ au 15 janvier 2001. Après dix jours d'accalmie, de nouvelles précipitations généralisées touchent la Bretagne entre le 31 décembre et le 5 janvier. Les cumuls atteignent $200 \mathrm{~mm}$ en certains endroits. L'ensemble des cours d'eau bretons est en crue. Ceux du Finistère connaissent deux pointes de crues de forte intensité le $1^{\text {er }}$ et le 5 janvier 2001. En Morbihan, elles surviennent entre le 5 janvier matin et le 6 au soir. Les périodes de retour dépassent ici les 20 ans et atteignent régulièrement les 50 ans, voire 100 ans sur l'Arz à Morlac. Même chronologie, avec des amplitudes un peu moindre dans les Côtes-d'Armor (10<T<20 ans), sauf sur l'Arguenon ( $T \approx 50$ ans). En Ille-et-Vilaine, les débits sont plus élevés que ceux enregistrés les mois précédents (en aval de Rennes, $T \approx 20$ ans et parfois 50 ans). A Redon, la combinaison des crues de l'Oust et de la Vilaine s'est traduite par de nombreux dommages aux habitations et aux entreprises implantées dans la zone d'activités.

Inondations du 24 au 28 janvier 2001. A partir du 24 janvier, un flux d'ouest sud-ouest particulièrement actif génère des épisodes pluvieux d'intensité moyenne pendant près de 48 heures. Les rivières entrent de nouveau en crue jusqu'au 28 janvier.

Les inondations de l'hiver 2000-2001 resteront marquantes avant tout par leur durée exceptionnelle. Aucune victime n'est à déplorer mais 1193 personnes ont été évacuées sur la région, et plus de 2600 habitations inondées. Les pertes matérielles et économiques sont évaluées à l'époque à plus d'un milliard de francs : 430 millions de francs pour les particuliers, 290 pour les entreprises, 35 pour l'agriculture, 290 pour les Collectivités et 1'Etat.
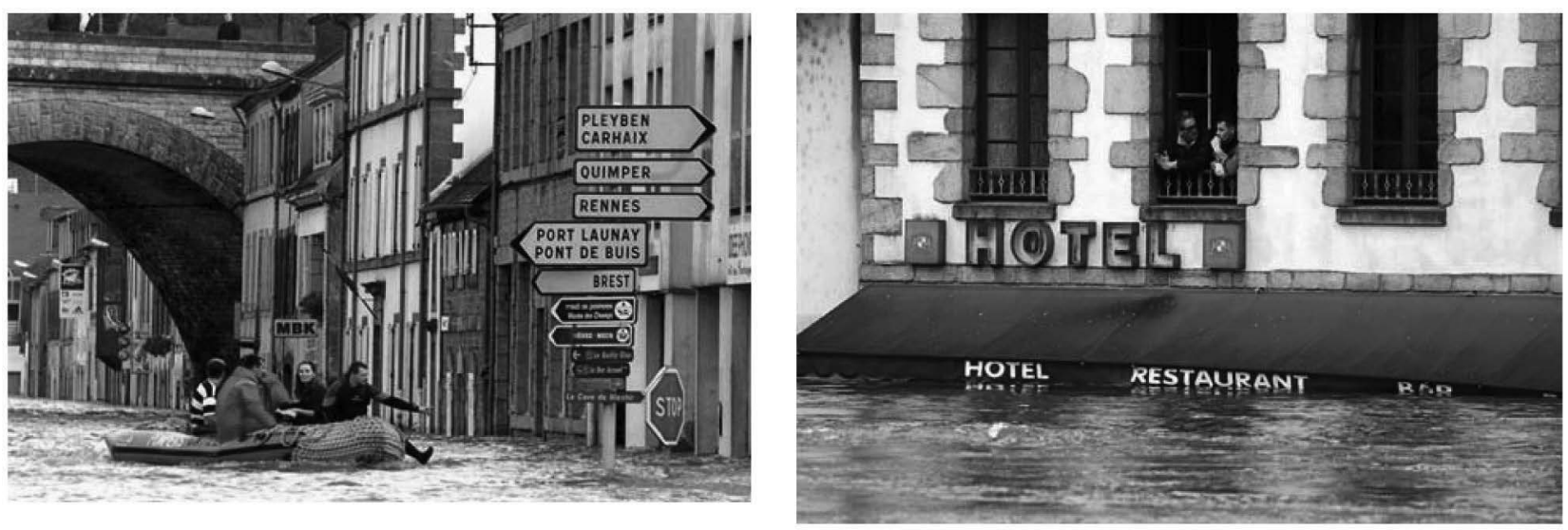

Figure 8 : Inondations du 12 décembre 2000 : (a) à Chateaulin ; (b) à Quimperlé (sources : Ouest-France et www.quimpermaville.com). 


\section{III.9. Remontées de nappe et inondations} de mars-avril 2001 dans le Nord de la France

Les inondations par remontée de nappe de mars-avril 2001 sont la conséquence d'une année 2000 particulièrement humide et d'une période de pluie exceptionnelle entre octobre 2000 et avril 2001. On relève sur cette période des cumuls pluviométriques supérieurs à $1000 \mathrm{~mm}$ sur près de la moitié des départements français, avec des valeurs de près du double de la moyenne climatologique sur la Normandie et la Picardie. La remontée du niveau de la nappe de la craie est exceptionnelle (plus fort niveau enregistré depuis les trente dernières années). En Haute-Normandie la nappe est remontée de plus de 20 mètres.

La crue de la Somme est remarquable tant par son ampleur que par sa durée. Les premières zones sont inondées fin février par les premiers affleurements de la nappe. Tout s'accélère fin mars. La submersion du lit majeur de la Somme commence. Chaque jour la conjugaison des débordements du fleuve et de ses affluents, des remontées de nappes et des ruissellements du bassin versant, inonde des superficies de plus en plus vastes. A la mi-avril, 155 communes sont touchées. De nombreuses zones urbanisées sont durement impactées. A Abbeville, on mesure, en certains endroits, plus de deux mètres d'eau. La gare est hors d'usage. La crue atteint son paroxysme mi-avril, avant de décroître très lentement ensuite. Le véritable retour à la normale ne sera effectif qu'au début de l'été. Une grande partie de la Haute-Normandie est également touchée, avec une centaine de communes concernées dans l'Eure et une trentaine en Seine-Maritime. Le bassin de la Seine ne connaît pas de crue d'intensité exceptionnelle, mais les débits restent soutenus pendant plusieurs mois. A Paris, le débit est resté au-dessus de $700 \mathrm{~m}^{3} / \mathrm{s}$ pendant 71 jours, ce qui correspond approximativement au seuil de vigilance de la station de ParisAusterlitz. Les voies sur berges sont fermées du 13 mars au 5 avril, puis du 9 avril au 12 mai. La navigation sur la Seine est interrompue entre le 17 mars et le 2 avril. Un grand nombre d'habitations sont inondées dans le bassin de l'Oise.

Au final sur le département de la Somme, plus de 3400 maisons sont inondées, dont 739 évacuées et 32 vouées à la destruction. Plus de 1100 personnes sont relogées (pour plusieurs semaines à plusieurs mois). Deux cents entreprises et 450 exploitations agricoles sont sinistrées. Les dommages s'élèvent à environ 150 millions d'euros, concentrés principalement sur les communes d'Abbeville, Fontaine-sur-Somme, Mareuil-Caubert, Amiens, Cagny, Camon et Pont-Rémy. Quelques 108 communes bénéficieront du dispositif CAT-NAT.

\section{III.10. Tempête Xynthia du 27-28 février 2010}

Les 27 et 28 février 2010, le littoral atlantique est frappé par la tempête Xynthia. La dépression, formée au milieu de
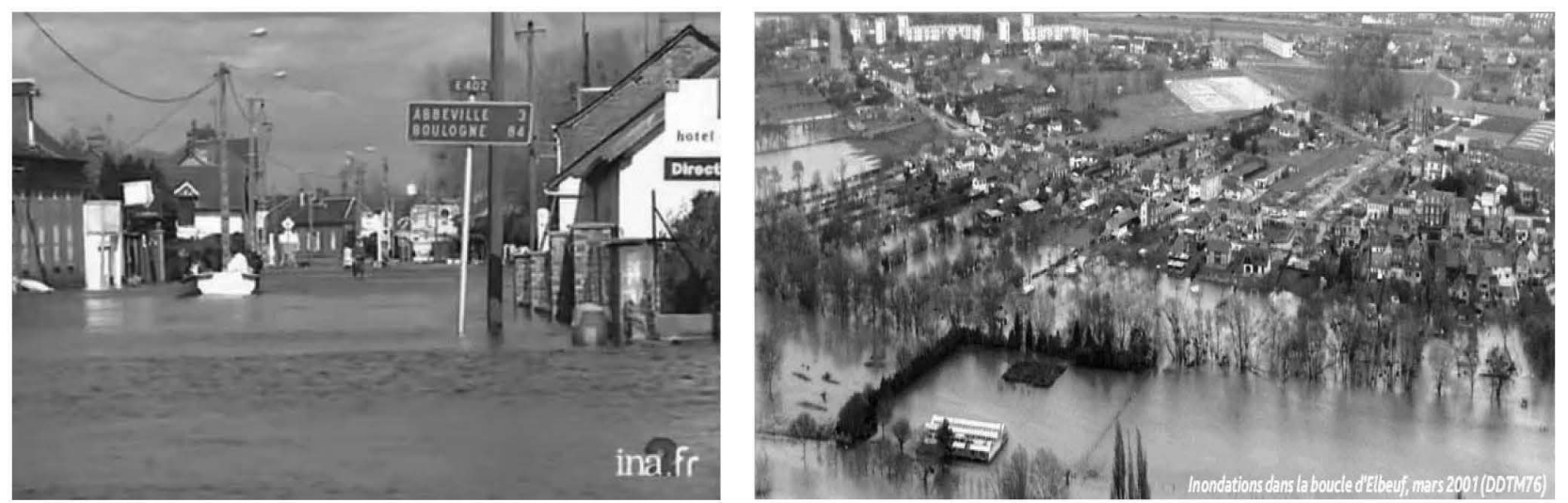

Figure 9 : Inondations de mars-avril 2001 : (a) Mareuil-Caubert, en périphérie d'Abbeville ; (b) dans la boucle d'Elbeuf en Haute-Normandie (sources : ina.fr et DDTM 76).

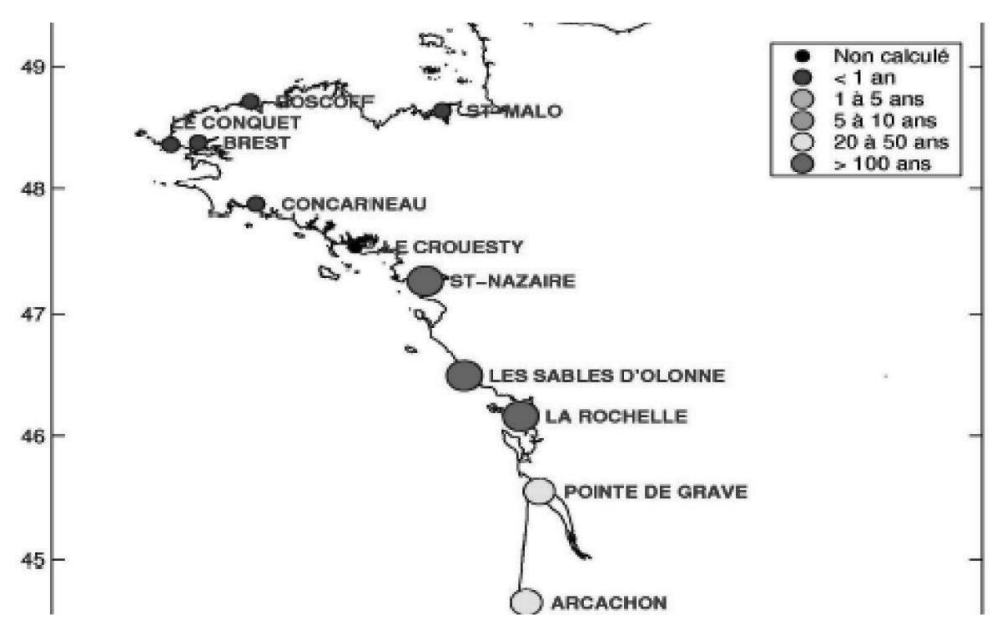

Figure 10 : Estimation des périodes de retour des hauteurs de pleine mer observées lors du passage de la tempête Xynthia en février 2010 (source: SHOM). 
l'Océan Atlantique au niveau du tropique du Cancer, évolue en tempête en remontant en direction des côtes européennes, avec une trajectoire atypique. L'intensité des deux tempêtes de décembre 1999 n'est pas atteinte, mais on relève tout de même des rafales de vent voisines de $160 \mathrm{~km} / \mathrm{h}$ sur le littoral et de 120 à $130 \mathrm{~km} / \mathrm{h}$ dans l'intérieur des terres. La houle provoquée par les vents, avec des vagues de six à sept mètres au large, s'ajoute à une élévation du niveau de la mer de grande ampleur qui trouve son origine dans la concomitance de Xynthia avec les grandes marées d'équinoxes (coefficient de marée de 102-108 à la pointe de Grave) et de son passage sur le littoral à l'heure de la pleine mer. Les surcotes de pleine mer les plus fortes sont observées à Saint-Nazaire et La Rochelle (resp. 1.16 et $1.53 \mathrm{~m}$ ). Les submersions touchent la totalité du littoral atlantique avec une intensité variable. Les phénomènes les plus importants sont localisés sur les côtes vendéennes entre la Rochelle et Saint Nazaire ( $T>100$ ans) et en Charente-Maritime. Au total, la tempête Xynthia balaye une large bande du territoire allant de la Charente-Maritime aux Ardennes, et provoque le décès de 53 personnes (dont 29 en Vendée et 11 en CharenteMaritime) et d'importants dégâts matériels. Leur montant est évalué à 1.5 milliard d'euros pour les assureurs.

\section{PERSPECTIVES DE VALORISATION DE L'ENQUÊTE 2011}

La notion d'événements extrêmes trouve toute sa pertinence quand elle peut s'appuyer sur une chronique d'événements bien décrits. Un des premiers constats de l'enquête 2011 est qu'on ne disposait à ce jour que d'une vision assez partielle de la réalité effective de ces événements remarquables à l'échelle du territoire national. Les causes sont diverses (pratiques et structurelles) en rapport avec la disponibilité et la capacité de mobilisation de la documentation depuis l'échelon local (département) jusqu'à l'échelon national. Les synthèses, type monographie, sont très rares. On dispose souvent de descriptions partielles et non validées, le tout conservé de manière aléatoire. Le travail de thèse en cours de Martin Boudou (2012-2015) va permettre d'approfondir la connaissance de ces événements remarquables d'inondation, et une grille de lecture commune a pu être mise au point, en combinant trois familles de critères, liés à l'extension spatiale, l'intensité et la gravité de l'inondation (Boudou et al., 2013). Le travail engagé dans le cadre de la DI vise justement à proposer une démarche globale de capitalisation à long terme de l'information sur les inondations. La BDHI pourrait ainsi constituer une référence pour tous les acteurs de la prévention, et participer dès lors activement à l'entretien de la mémoire et au développement de la culture du risque. Des collaborations fructueuses sont attendues dans l'avenir entre scientifiques, opérateurs techniques de l'environnement et gestionnaires autour de ce projet.

\section{REMERCIEMENTS}

Cette première phase d'enquête documentaire a très largement mobilisé les services de l'Etat en charge de la prévention des inondations en métropole et dans les DOM (DREAL, DDT, SPC, SN, etc.). Nous les remercions ici pour leur collaboration dans une démarche qui à plusieurs reprises a dû s'adapter aux contraintes du calendrier de la Directive Inondation et à celles de sa mise en œuvre pratique. La DGPR, qui pilote l'ensemble du travail sur la DI, a su être réactive pour mobiliser les énergies des différents services. D'autres acteurs publics ont été sollicités ponctuellement et le seront sans doute plus largement lors des prochaines étapes : EPTB, Archives Nationales et départementales, Météo France. Nous remercions également les équipes universitaires de géographes et historiens de Montpellier, Toulouse, Strasbourg, Caen, et Lyon qui à un titre ou un autre nous ont aidé à la réalisation de cette première phase.

\section{REFERENCES ET CITATIONS}

Compte tenu de la nature du travail (synthèses à partir d'études antérieures), nous ne présentons ci-dessous qu'une brève sélection des publications et sources mobilisées.

\section{Documents publiés}

Ambroise-Rendu M. (1910) - Paris inondé

Antoine J.M., Desailly B., Gazelle F. (2001) - Les crues meurtrières, du Roussillon aux Cévennes. Annales de Géographie. 622

Baulig H. (1950) - Les inondations de décembre 1947 (Publications du Comité consultatif météorologique du Bas-Rhin). Annales de l'Institut de physique du globe de Strasbourg, Géophysique, Strasbourg, Imprimerie Alsacienne. $\mathbf{V ( 3 )}: 12$ pages, cartes

Belgrand (1875) - Hydrologie du bassin de la Seine. 2 : 1875

Boudou M., Ceeur D., Lang M., Vinet F. (2013) - Grille de lecture pour la caractérisation d'événements remarquables d'inondation en France : exemple d'application pour la crue de mars 1930. Colloque « Dynamiques environnementales, politiques publiques et pratiques locales : quelles interactions?", Toulouse, 4-7 juin

ChAmpion M. (2001) - Les inondations en France du VI $I^{e}$ siècle à nos jours. Cemagref Editions, Réédition ouvrage de 1858. 3000 p., 6 tomes

Diren D’ile-De-France, Bassin Seine-Normandie, Dreal Basse Normandie (2005) - Schéma directeur de prévision des crues du bassin Seine-Normandie2005 62p

DOUVINET J. (2008) - Les bassins versants sensibles aux « crues rapides » dans le Bassin Parisien - Analyse de la structure et de la dynamique de systèmes spatiaux complexes Thèse de Doctorat, Université de Caen, 2008

LORION D. (2000) - Inondation et aménagements à l'île de la Réunion - Université de ParisX. 529p

Martin B. Ansel R., Guerrouah O., Vitoux M.C., With L., Drescher A., Glaser R., Himmelsbach I. Et J. Schönbein (2011) - Géohistoire critique de la crue de janvier 1910 dans le fossé Rhénan (Alsace / Pays de Bade). La Houille Blanche. 1 : $62-68$

Nouaillhac-Pioch \& Maillet (1910) - Monographie de la crue de janvier. 1910

PARDE M. (1930) - La crue catastrophique de mars 1930 dans le Sud-Ouest de la France

PARDE M. (1941) - Averses et crues fantastiques dans le Roussillon en octobre 1940

VINET F. (2003) - Crues et inondations dans la France méditerranéenne. Les crues torrentielles des 12 et 13 novembre 1999 (Aude, Tarn, Pyrénées-Orientales, Hérault), Editions du Temps

\section{Documents et sources non publiés}

Archives Nationales, Dossiers Inondations (Bassins, Seine, Loire, Rhone, Garonne) F : 14 
BRGM (2011) - Contribution au recensement des submersions marines historiques liées aux tempêtes sur le littoral français métropolitain

CARIOLET (J.-M.) - Inondation des côtes basses et risques associés en Bretagne. Thèse, Université de Bretagne occidentale, 2010

Cete Mediterranee (2010) - Analyse historique des séries marégraphiques du Golfe du Lion, tempêtes, crues, raz-de-marée, tsunamis et remontées du niveau marins

Dreal Reunion (2010) - Rapport Données Historiques 2010 Synthèse des événements : HYACINTHE cyclone tropical (16 au 27 janvier 1980)
DGPR/SRT/BARPI (2009) - Rupture d'un Barrage, le 2 Decembre 1959, Malpasset, France

Dreal Languedoc-Roussillon (2010) - Analyse historique des séries marégraphiques du Golfe du Lion

Collectif (2001) - Rapport interministériel sur les crues de 2000 et 2001

\section{Sites web}

www.crue1910.fr ; http ://pluiesextremes.meteo.fr ; www.tsunamis.fr/. 